\title{
Spontaneous Transverse Response and Amplified Switching in Superconductors with Honeycomb Pinning Arrays
}

\author{
C. Reichhardt and C.J. Olson Reichhardt \\ Theoretical Division, Los Alamos National Laboratory, Los Alamos, New Mexico 87545
}

(Dated: November 6, 2018)

\begin{abstract}
Using numerical simulations, we show that a novel spontaneous transverse response can appear when a longitudinal drive is applied to type-II superconductors with honeycomb pinning arrays in a magnetic field near certain filling fractions. This response is generated by dynamical symmetry breaking that occurs at fields away from commensurability. We find a coherent strongly amplified transverse switching effect when an additional transverse ac drive is applied. The transverse ac drive can also be used to control switching in the longitudinal velocity response. We discuss how these effects could be used to create new types of devices such as current effect transistors.
\end{abstract}

PACS numbers: $74.25 . \mathrm{Qt}$

There have been extensive studies on superconducting systems with patterned pinning arrays which show commensurability effects when the vortex density matches the pinning site density [1, 2, 3, 4]. Various properties of the vortex transport can be controlled by adjusting the shape and geometry of the pinning arrays, producing vortex channeling [5], dynamical transitions [6, 7], fluxon ratchets [8], reversible ratchets [9, 10], and vortex cellular automata [1]. These studies indicate that vortices interacting with patterned substrates may lead to a new field of fluxtronics or microelectronic devices based on the controlled motion of vortices. Further, vortices interacting with periodic pinning arrays also exhibit a number of collective dynamic behaviors which are important to the broader field of nonequilibrium physics.

Few studies have been performed on vortex dynamics in honeycomb pinning arrays since it was assumed that the vortex behavior in this pinning geometry would be similar to that found in square and triangular pinning arrays. In this Letter we demonstrate that in fact, honeycomb pinning arrays produce new types of phenomena that do not occur in triangular or square pinning arrays. In particular, an effective dimerization or higher order $n$-merization of the interstitial vortices in the honeycomb pinning arrays for certain field ranges results in a novel spontaneous transverse response (STR) to a longitudinal drive. Normally, an applied current produces a perpendicular Lorentz force on superconducting vortices, which move and generate a voltage drop parallel to the current. We show that the honeycomb system can also produce a voltage drop perpendicular to the applied current. For magnetic fields away from commensuration, the vortex flow dynamically organizes to a STR state. At the matching fields, the STR arises due to symmetry breaking in the ground state. The vortex $n$-mer states are very similar to the colloidal molecular crystal states studied for colloids interacting with periodic optical trap arrays [12]. We specifically consider vortices in superconductors; however, we expect similar phases to occur whenever there is a spontaneous symmetry breaking of effective $n$-mer states for particles on periodic substrates.

We simulate a two-dimensional system containing $N_{v}$ vortices and $N_{p}$ pinning sites with periodic boundary conditions in the $x$ and $y$ directions. The vortex density is $B=N_{v} \phi_{0} / L^{2}$, where $L=24 \lambda$ is the system size in units of the penetration depth $\lambda$ and $\phi_{0}=h / 2 e$ is the elementary flux quantum. The motion of a single vortex is given by the following overdamped equation:

$$
\eta \frac{d \mathbf{R}_{i}}{d t}=\mathbf{F}_{i}^{v v}+\mathbf{F}_{i}^{p}+\mathbf{F}^{d c}+\mathbf{F}^{a c}+\mathbf{F}_{i}^{T}
$$

Here $\mathbf{R}_{i}$ is the location of vortex $i, \eta=\phi_{0}^{2} d / 2 \pi \xi^{2} \rho_{N}$ is the damping constant, $d$ is the thickness of the superconducting sample, $\xi$ is the coherence length, and $\rho_{N}$ is the normal state resistivity. The pairwise vortex-vortex interaction force is $\mathbf{F}_{i}^{v v}=\sum_{i \neq j}^{N_{v}} f_{0} K_{1}\left(R_{i j} / \lambda\right) \hat{\mathbf{R}}_{i j}$ where $K_{1}$ is the modified Bessel function, $R_{i j}=\left|\mathbf{R}_{i}-\mathbf{R}_{j}\right|, \hat{\mathbf{R}}_{i j}=$ $\left(\mathbf{R}_{i}-\mathbf{R}_{j}\right) / R_{i j}$, and $f_{0}=\phi_{0}^{2} /\left(2 \pi \mu_{0} \lambda^{3}\right)$. The pinning force $\mathbf{F}_{i}^{p}$ arises from parabolic traps of radius $r_{p}=0.3 \lambda$ and strength $f_{p}=1.0 f_{0}$ arranged in a honeycomb lattice with $\mathbf{F}_{i}^{p}=-\sum_{k=1}^{N_{p}} f_{p} R_{i k} r_{p}^{-1} \Theta\left(\left(R_{p}-R_{i k}\right) / \lambda\right) \hat{\mathbf{R}}_{i k}$. Here $R_{i k}=$ $\left|\mathbf{R}_{i}-\mathbf{R}_{k}\right|$ is the distance between vortex $i$ and pin $k$, $\hat{\mathbf{R}}_{i k}=\left(\mathbf{R}_{i}-\mathbf{R}_{k}\right) / R_{i k}$, and $\Theta$ is the Heaviside step function. The dc longitudinal driving force $\mathbf{F}^{d c}=F^{d c} f_{0} \hat{\mathbf{R}}^{L}$, where we take the longitudinal direction $\hat{\mathbf{R}}^{L}$ to be $\hat{\mathbf{R}}=\hat{\mathbf{x}}$. This force mimics the Lorentz force created by an applied current. We also consider the effect of adding a transverse ac drive $\mathbf{F}^{a c}=F^{a c} f_{0} \sin (\omega t) \hat{\mathbf{R}}^{T r}$, where $\omega=10^{-7} f_{0} / \eta$ and $\hat{\mathbf{R}}^{T r}=\hat{\mathbf{y}}$ is the transverse direction. The thermal force $\mathbf{F}_{i}^{T}$ arises from Langevin kicks and has the properties $\left\langle F_{i}^{T}(t)\right\rangle=0$ and $\left\langle F_{i}^{T}(t) F_{j}^{T}\left(t^{\prime}\right)\right\rangle=2 \eta k_{B} T \delta_{i j} \delta\left(t-t^{\prime}\right)$. Vortex positions are initialized with simulated annealing.

In Fig. 1 we illustrate the vortex and pinning site positions in a sample with $F^{d c}=0, F^{a c}=0$, and $T=0$. At $B / B_{\phi}=2.0$, shown in Fig. $1(\mathrm{a}, \mathrm{b}, \mathrm{c})$, where $B_{\phi}$ is the field at which $N_{v}=N_{p}$, the large interstitial space of each plaquette in the honeycomb pinning lattice traps two interstitial vortices which form an effective rigid dimer with a director field that can point in one of three degenerate directions, as indicated by the dashed 

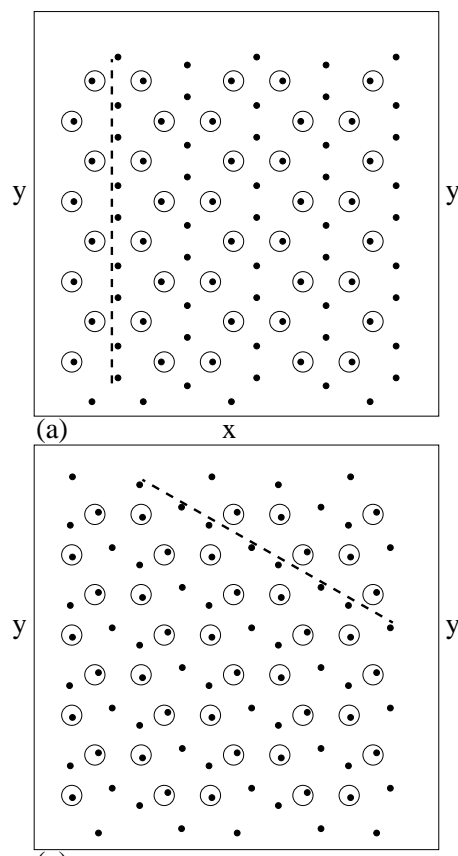

(c)
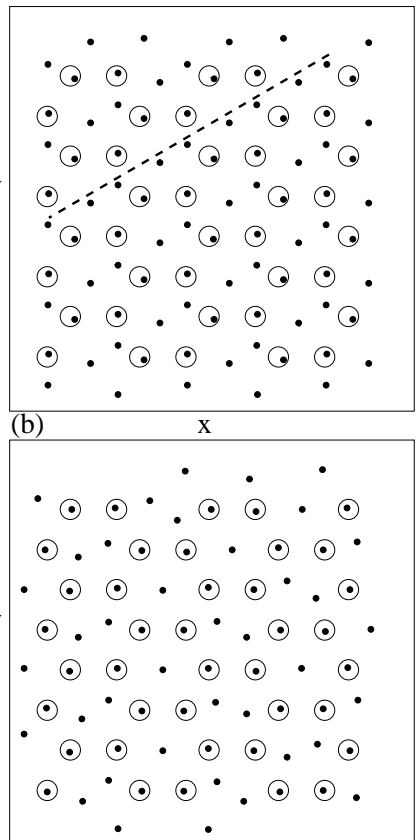

(d)

FIG. 1: Vortex positions (black dots) and pinning site locations (open circles) in a $20 \lambda \times 20 \lambda$ section of the sample at $F^{d c}=0 . \quad(\mathrm{a}, \mathrm{b}, \mathrm{c})$ : Three different realizations of $B / B_{\phi}=2.0$ with interstitial dimers aligned (a) in the $y$ direction; (b) at $+30^{\circ}$ to the $x$-direction; (c) at $-30^{\circ}$ to the $x$-direction. The dashed lines indicate the alignment direction. (d) $B / B_{\phi}=1.77$ with no overall dimer alignment.

lines. Neighboring dimers interact through an effective quadrupole interaction which causes the dimers to align into one of three degenerate ground states [13]. For fillings $1.5<B / B_{\phi}<2.0$ and $2.0<B / B_{\phi}<2.5$, the dimer alignment is disrupted and there is no global symmetry breaking in the ground state. Figure 1(d) shows an example at $B / B_{\phi}=1.77$ where the dimer ordering is lost.

We next apply a dc drive $F^{d c}$ in the longitudinal direction and measure the vortex velocity $\mathbf{v}$ to obtain the longitudinal velocity $V_{L}=\sum_{i}^{N_{v}} \mathbf{v}_{i} \cdot \hat{\mathbf{R}}^{L}$ and the transverse velocity $V_{T r}=\sum_{i}^{N_{v}} \mathbf{v}_{i} \cdot \hat{\mathbf{R}}^{T r}$. In Fig. 2(a) we plot $V_{L}$ and $V_{T r}$ versus $F^{d c}$ for the system in Fig. 1 at $B / B_{\phi}=2.0$. For $F^{d c}<0.14$ the system is pinned, while for $0.14 \leq F^{d c}<0.37$ there is a finite longitudinal velocity $V_{L}$ accompanied by a finite transverse response $V_{T r}$ that can be in either the positive $\left(V_{T r}^{+}\right)$or negative $\left(V_{T r}^{-}\right)$ transverse direction. The trajectories of the moving vortices for the $V_{T r}^{+}$and $V_{T r}^{-}$states are shown in Figs. 3(a) and (b), respectively. When $F^{d c}>F_{c}^{d c}$, where $F_{c}^{d c}$ is the critical drive at which vortices in the pinning sites begin to depin, the symmetry breaking is lost. At $B / B_{\phi}=2.0$, $F_{c}^{d c}=0.37$, and above this drive, $\left|V_{T r}\right|$ drops abruptly to zero and $V_{L}$ rapidly increases. The curve with $V_{T r}^{+}$was obtained by starting from the ground state in Fig. 1(a) at $T=0$ while the curve with $V_{T r}^{-}$was generated starting from the same ground state but applying a finite but
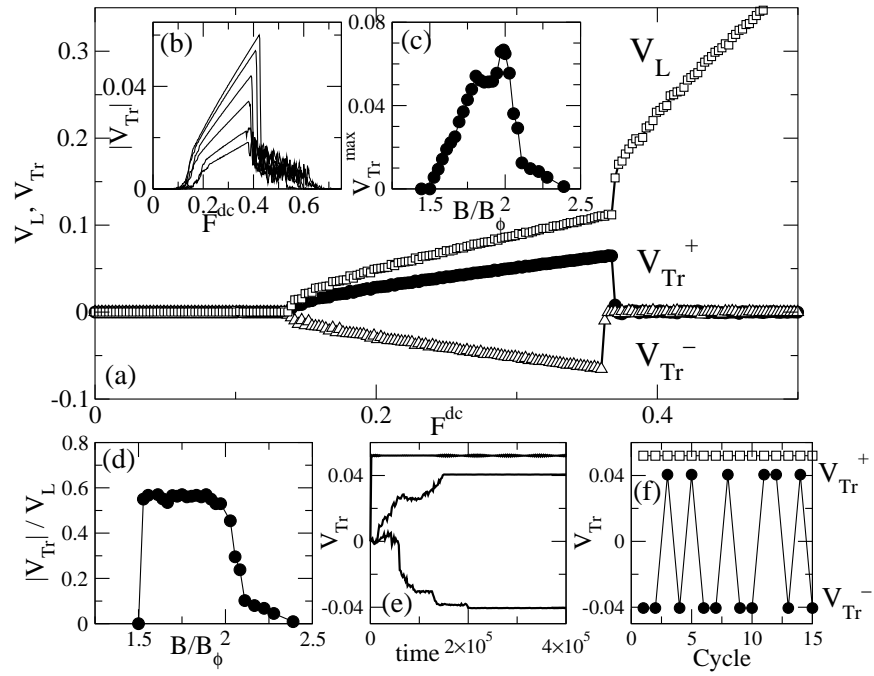

FIG. 2: (a) Transverse velocities $V_{T r}^{ \pm}$and longitudinal velocity $V_{L}$ versus $F^{d c}$ for the sample in Fig. 1 at $B / B_{\phi}=2.0$. (b) $\left|V_{T r}\right|$ vs $F^{d c}$ for the same system at $B / B_{\phi}=1.58,1.61,1.67$, $1.72,1.77$, and 1.81 , from bottom to top. (c) $V_{T r}^{\max }$ versus $B / B_{\phi}$. (d) $\left|V_{T r}\right| / V_{L}$ versus $B / B_{\phi}$ at fixed $F^{d c}=0.225$. (e) Time series of $V_{T r}$ for $F^{d c}=0.3$ at $B / B_{\phi}=2.0$ (top curve) and $B / B_{\phi}=1.81$ (two lower curves). (f) $V_{T r}$ during consecutive cycles of $F^{d c}$ from 0 to $F^{d c}=0.3$ at $B / B_{\phi}=2.0$ (open squares) and $B / B_{\phi}=1.81$ (filled circles).

small temperature $T=0.2 T_{m}$, where $T_{m}$ is the vortex lattice melting temperature. If we repeat the finite temperature simulation with different random seeds, we are equally likely to observe $V_{T r}^{+}$or $V_{T r}^{-}$. If the initial ground state already has a global symmetry preferred positive or negative orientation with respect to the transverse direction, as in Figs. 1(b) and (c), the transverse response is in the same direction indicated by the dashed lines in Fig. 1(b,c). The appearance of a transverse velocity at $B / B_{\phi}=2.0$ is not a manifestation of a dynamical symmetry breaking since the dimer alignment symmetry is already broken as in Fig. 1. In contrast, for the fields $1.5<B / B_{\phi}<2.0$ and $2.0<B / B_{\phi}<2.5$ there is no global symmetry breaking in the ground states; however, a dynamical symmetry breaking occurs when the vortices organize into a dynamical phase resembling the states in Figs. 3(a,b) after a transient period of time during which the vortices move in both the $V_{T r}^{+}$and $V_{T r}^{-}$directions.

In Fig. 2(b) we plot $\left|V_{T r}\right|$ as a function of $F^{d c}$ at $B / B_{\phi}=1.58,1.61,1.67,1.72,1.77$, and 1.81 showing the appearance of the dynamical broken symmetry state. In Fig. 2(e) we plot $V^{T r}$ vs time for $F^{d c}=0.3$ at $B / B_{\phi}=2.0$ and $B / B_{\phi}=1.81$, where the latter curves were generated from samples in slightly different initial states. At $B / B_{\phi}=2.0$, the vortices immediately move in the broken symmetry direction of the ground state, which in this case is $V_{T r}^{+}$shown in Fig. $1(\mathrm{~b})$. For $B / B_{\phi}=1.81$, the initial motion is symmetric and only develops into a 

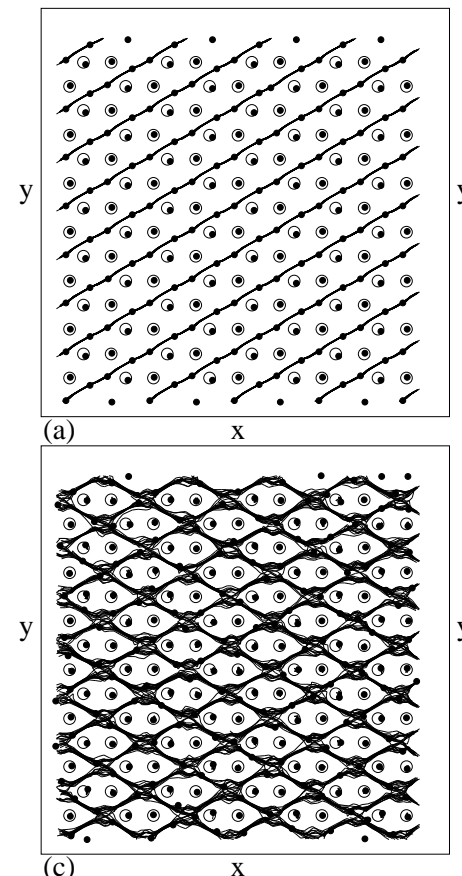

(c)

$\mathrm{x}$

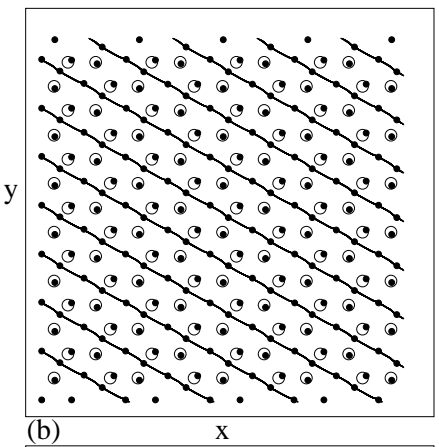

(b)

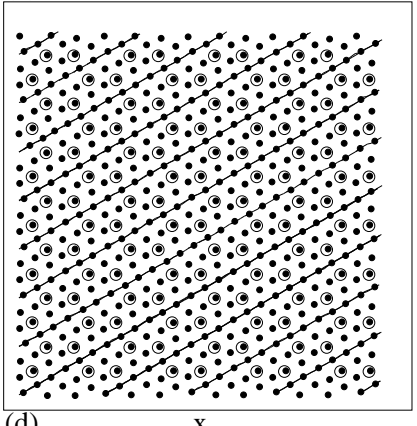

(d)
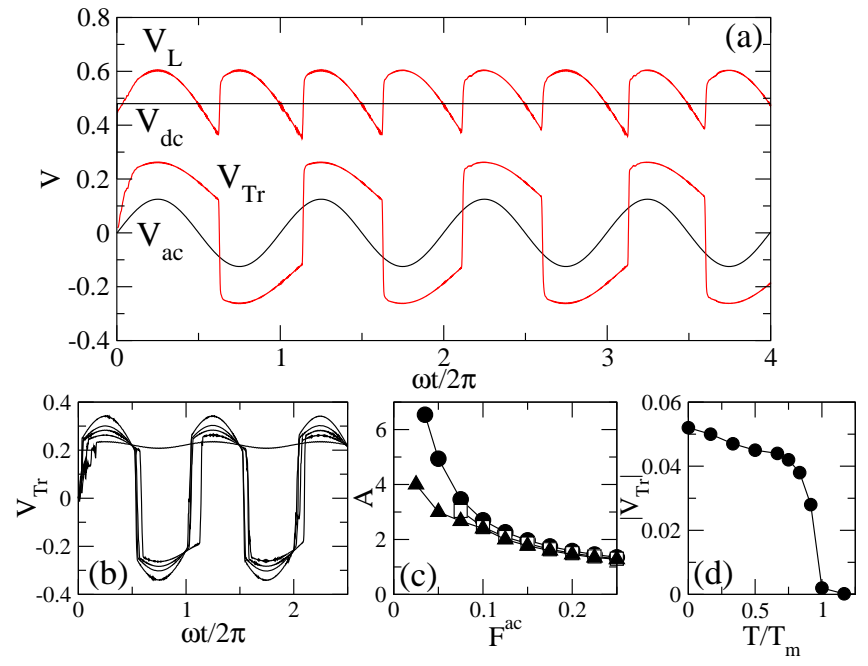

FIG. 4: (a) $V_{T r}(t)$ (thick lower line) and $V_{L}(t)$ (thick upper line), with the analytic values of $V_{a c}(t)$ (thin lower line) and $V_{d c}(t)$ (thin upper line) shown for comparison. $V_{L}$ and $V_{d c}$ have been shifted up by 0.2 for presentation purposes. Time is given in units of $2 \pi / \omega$. Here $B / B_{\phi}=2.0, F^{a c}=0.125$, and $F^{d c}=0.28$. (b) Time series of $V_{T r}$ for a system with $B / B_{\phi}=$ $1.77, F^{d c}=0.28$, and varied ac drive amplitude of $F^{a c}=0.15$, $0.1,0.075,0.05,0.035$, and 0.02 , from top to bottom on the positive response side. (c) The amplification factor $A$ versus $F^{a c}$ for samples at $F^{d c}=0.28$ with $B / B_{\phi}=1.61$ (filled circles), 2.0 (open squares), and 2.11 (filled triangles). (d) $\left|V_{T r}\right|$ vs $T / T_{m}$ at $B / B_{\phi}=2.0$ and $F^{d c}=0.3$.

elongated trimers can form, as illustrated in Fig. 3(d). This is accompanied by spontaneous dynamical symmetry breaking over a range of fields. We have also considered the role of temperature. Previous work [13] indicated that the interstitial vortices melt at a well defined temperature $T_{m}$; using parameters appropriate for $\mathrm{Nb}$ crystals gives $T_{m} \approx 8.5 \mathrm{~K}$. We find that our results are robust for all $T<T_{m}$. In Fig. 4(d) we plot $\left|V_{T r}\right|$ vs $T / T_{m}$ at $B / B_{\phi}=2.0$ and $F^{d c}=0.3$, showing that $V_{T r}$ drops sharply with increasing $T$ just below $T / T_{m}=1.0$.

Since the dynamical symmetry-broken states are bistable, it is natural to ask whether it is possible to induce a switching behavior between the two states. To address this, we consider the effect of adding a transverse oscillating drive $F^{a c}$ to a system with $B / B_{\phi}=2.0$ moving under a fixed longitudinal drive $F^{d c}=0.28$. Experiments have already been conducted in superconductors with periodic pinning arrays where two orthogonal driving currents were simultaneously applied and the transverse and longitudinal responses were simultaneously measured [17]. In Fig. 4(a) we plot the time series $V_{T r}(t)$ and $V_{L}(t)$ in a system with $F^{a c}=0.125$. Here the ac drive induces a periodic switching between the positive and negative transverse response states $V_{T r}^{+}$ and $V_{T r}^{-}$. We find that this abrupt switching effect per-

sists for $1.5<B / B_{\phi} \leq 2.0$. Similar switching appears

The same type of symmetry breaking flow occurs higher commensurate states such as $B / B_{\phi}=4.5$ where 
over a wide range of ac driving amplitudes, as illustrated in Fig. 4(b) where we plot $V_{T r}(t)$ at $B / B_{\phi}=1.77$ and $F^{d c}=0.28$ for different values of $F^{a c}$. We observe a novel response in the longitudinal direction in which the value of $V_{L}(t)$ varies by up to $50 \%$ during each ac drive period. There is also a switching effect in $V_{L}(t)$ which accompanies each switch in $V_{T r}(t)$. This phenomenon is reminiscent of the current effect transistor found in transversely driven charge density waves where a transverse force can be used to control the longitudinal response [15].

There is an amplification of the transverse ac response $V_{T r}(t)$ over the value expected based only on the magnitude of the ac input driving signal. To illustrate this, in Fig. 4(a) we plot $V_{a c}(t) \equiv N_{m} F^{a c}(t) / \eta$, the transverse ac velocity produced when the $N_{m}=N_{v}-N_{p}$ interstitial vortices move only in response to the ac driving force, along with $V_{d c} \equiv N_{m} F^{d c} / \eta$, the longitudinal velocity of the interstitial vortices under only the dc drive. Fig. 4(a) indicates that $\left|V_{T r}(t)\right|>\left|V_{a c}(t)\right|$. The relative amplification $V_{T r}(t) / V_{a c}(t)$ can be increased by lowering $F^{a c}$; however, for a finite $F^{d c}$ there is a threshold value $F_{c}^{a c}$ below which the system no longer switches between $V_{T r}^{+}$ and $V_{T r}^{-}$. For example, in Fig. 4(b), $F^{a c}=0.02$ is below the threshold $F_{c}^{a c}$, and thus the response stays locked in the $V_{T r}^{+}$direction. We quantify the amplification of the transverse ac response using $A=V_{T r} \eta /\left(N_{m} F^{a c}\right)$, where $V_{T r}$ is the amplitude of the transverse response, such that for $A=1.0$, there is no amplification. The plot of $A$ vs $F^{a c}$ in Fig. 4(c) for $B / B_{\phi}=1.61,2.0$, and 2.11 shows that for a fixed dc drive, the amount of ac amplification that occurs depends on both $F^{a c}-F_{c}^{a c}$ and $F_{c}^{d c}-F^{d c}$. The closer the ac and dc drives are to the respective critical thresholds, the larger the amplification. Since $F_{c}^{d c}$ is maximized at $B / B_{\phi}=2.0$, for fixed $F^{d c}=0.28$ a larger amplification can be obtained at $B / B_{\phi} \neq 2.0$ than at $B / B_{\phi}=2.0$, as shown in Fig. 4(c). For the parameters used in Fig. 4, the frequencies for a typical superconductor are between 10 and $30 \mathrm{~Hz}$. If $\omega$ is increased, the sharp switching response can still be achieved by increasing $F^{a c}$ until $\omega \sim 20$ to $60 \mathrm{KHz}$, at which point $F_{c}^{a c}$ crosses above the depinning threshold for the pinned vortices and the dynamical symmetry breaking is completely lost.

Many of the switching features, including bistability in the transverse response and the abrupt switching effect induced by the ac drive, are analogous to semiconductors and may be useful for creating logic devices. The strong coupling between the longitudinal and transverse responses indicates that a transverse ac drive could be used to control longitudinal switching, while the amplification effect implies that very small ac inputs can induce large response changes in certain regimes. Our results should apply to any system of repulsively interacting particles confined by honeycomb pinning arrays. We have observed a similar dynamical symmetry breaking flow for colloids on triangular substrates [16]; however, the dynamical symmetry breaking for the vortex system presented here is much more robust and occurs for a considerably wider range of parameters and particle fillings.

In summary, we have demonstrated that vortices in superconductors with honeycomb pinning arrays can exhibit a novel transverse response when a longitudinal drive is applied for certain ranges of fields where the vortices in the large interstitial regions of the pinning array form effective dimer or higher order $n$-mer states. At commensurate fields, the symmetry is broken in the ground state, while at incommensurate fields, there is a dynamical symmetry breaking. If a transverse ac drive is added to the system, a pronounced transverse switching response occurs, as well as an amplification of the transverse ac signal. There is a threshold ac drive required to induce the switching which becomes very small when the longitudinal drive is close to the value above which the transverse response disappears. The transverse ac drive can be used to modulate the longitudinal response of the system as well. We discuss how this effect may be useful for creating new types of fluxon based devices.

This work was carried out under the auspices of the NNSA of the U.S. DoE at LANL under Contract No. DE-AC52-06NA25396.

[1] M. Baert et al,, Phys. Rev. Lett. 74, 3269 (1995); K. Harada et al., Science 274, 1167 (1996); J.I. Martín et al., Phys. Rev. Lett. 79, 1929 (1997); A.N. Grigorenko et al., Phys. Rev. Lett. 90, 237001 (2003).

[2] G. Karapetrov et al., Phys. Rev. Lett. 95, 167002 (2005).

[3] C. Reichhardt, C.J. Olson, and F. Nori, Phys. Rev. B 57, 7937 (1998); C.J. Olson Reichhardt, A. Libál, and C. Reichhardt, Phys. Rev. B 73, 184519 (2006).

[4] G.R. Berdiyorov, M.V. Milosevic, and F.M. Peeters, Phys. Rev. B 74, 174512 (2006).

[5] M. Velez et al., Phys. Rev. B 65, 104511 (2002).

[6] C. Reichhardt, G.T. Zimányi, and N. Grønbech-Jensen, Phys. Rev. B 64, 014501 (2001).

[7] R. Surdeanu et al., Europhys. Lett. 54, 682 (2001).

[8] C.S. Lee et al., Nature (London) 400, 337 (1999).

[9] J.E. Villegas et al., Phys. Rev. B 71, 024519 (2005).

[10] C.C. de Souza Silva et al., Nature (London) 440, 651 (2006).

[11] M.B. Hastings, C.J. Olson Reichhardt, and C. Reichhardt, Phys. Rev. Lett. 90, 247004 (2003).

[12] C. Reichhardt and C.J. Olson, Phys. Rev. Lett 88, 248301 (2002); M. Brunner and C. Bechinger, ibid. 88, 248302 (2002); A. Sarlah, E. Frey, and T. Franosch, Phys. Rev. E 75, 021402 (2007).

[13] C. Reichhardt and C.J. Olson Reichhardt, Phys. Rev. B 76, 064523 (2007).

[14] T.C. Wu et al., J. Appl. Phys. 97, 10 B102 (2005).

[15] L. Radzihovsky and J. Toner, Phys. Rev. Lett. 81, 3711 (1998).

[16] C. Reichhardt and C.J. Olson Reichhardt, Europhys. Lett. 68, 303 (2004).

[17] J.E. Villegas et al., Phys. Rev. B 68, 224504 (2003); 72, 064507 (2005). 